\title{
Estudo de melanoma da coróide no Hospital Regional de São José - Santa Catarina
}

\author{
Study on choroidal melanoma at the Oph thalmology unit of the Regional \\ Hospital of São José - Santa Catarina
}

\author{
Astor Grumann Júnior ${ }^{1}$ \\ Augusto Adam Neto ${ }^{2}$ \\ Gina Carriero Lima $^{3}$ \\ Franciele Vergini ${ }^{4}$ \\ Thiago de Azevedo Borges de Souza ${ }^{5}$
}

${ }^{1}$ Doutor em Oftalmologia pela Universidade Federal de Minas Gerais - UFMG - Belo Horizonte (MG) - Brasil, Supervisor da Residência Médica de Oftalmologia do Hospital Regional de São José - São José (SC) - Brasil, Professor da Disciplina de Oftalmologia da Universidade do Sul de Santa Catarina - UNISUL - Tubarão (SC) - Brasil.

2 Doutor em Oftalmologia pela Universidade Federal de Santa Catarina - UFSC, Professor Titular da Disciplina de Oftalmologia da UFSC, Professor da Disciplina de Oftalmologia da UNISUL - Tubarão (SC) - Brasil.

3 Professora da Disciplina de Oftalmologia da UNISUL - Tubarão (SC) - Brasil.

${ }^{4}$ Residente do Serviço de Oftalmologia do Hospital Regional de São José - São José (SC) - Brasil.

5 Acadêmico do Curso de Medicina da UNISUL - Tubarão (SC) - Brasil.

Endereço para correspondência: Thiago de Azevedo Borges de Souza. Rua Florianópolis, 95 - Tubarão (SC) CEP 88705-080

Recebido para publicação em 04.05.2005

Versão revisada recebida em 03.01.2006

Aprovação em 12.01.2006

Nota Editorial: Depois de concluída a análise do artigo sob sigilo editorial e com a anuência da Dra. Vera Regina Cardoso Castanheira sobre a divulgação de seu nome como revisora, agradecemos sua participação neste processo.

\begin{tabular}{l} 
RESUMO \\
\hline Objetivo: Descrever as características dos pacientes com melanoma de \\
coróide diagnosticados e tratados no Serviço de Oftalmologia do Hos- \\
pital Regional de São José - Santa Catarina, e apresentar os resultados. \\
Métodos: Análise de uma série de 24 pacientes diagnosticados com \\
melanoma maligno de coróide no período de $1^{\circ}$ de março de 1997 a 21 de \\
julho de 2003 , avaliando: idade, sexo, raça, sintomas e duração, métodos \\
diagnósticos, tratamento realizado, tamanho e tipo do tumor. Resulta- \\
dos: Não existiu diferença em relação ao sexo e a idade média dos \\
pacientes foi de $57,20 \pm 16,56$ anos. O tumor predominou em brancos \\
(83,33\%) e a baixa da acuidade visual foi o sintoma mais freqüente \\
(83,33\%), sendo o tempo médio do início da sintomatologia até atendi- \\
mento inicial de $152,12 \pm 114,00$ dias. A oftalmoscopia indireta e a ultra- \\
sonografia foram utilizadas em todos os pacientes para diagnosticar o \\
tumor. Todos os pacientes foram submetidos à enucleação, pois apre- \\
sentavam tumores grandes (maior diâmetro basal superior a 16 mm na \\
USG). O tipo mais freqüente foi o de células fusiformes (58,5\%) e o \\
tamanho médio dos tumores foi de $11,8 \pm 4,5$ mm de diâmetro basal. Dois \\
pacientes apresentaram metástase à distância. Conclusões: Todos os \\
pacientes foram diagnosticados em estágio avançado do tumor, pioran- \\
do o prognóstico visual e sistêmico e limitando as opções terapêuticas, \\
sendo por isso enucleados.
\end{tabular}

Descritores: Melanoma/diagnóstico; Neoplasias da coróide; Enucleação ocular

\section{INTRODUÇÃO}

Os melanomas oculares constituem-se na forma não cutâneos mais comum deste tipo histológico de tumor, porém são raros, se comparados aos melanomas da pele ${ }^{(1)}$. Foi estimado que os melanomas malignos intraoculares ocorram em 0,02 a $0,06 \%$ do total dos pacientes oftalmológicos dos Estados Unidos da América ${ }^{(2)}$. Sendo mais freqüentes na $6^{\underline{a}}$ década de vida e muito raro na $1^{\underline{a}}$ e $2^{\underline{a}}$ década $^{(3)}$. Ambos os sexos são igualmente afetados, acometendo com mais freqüência pacientes brancos, sendo raros em negros e asiáticos ${ }^{(1)}$. São, por via de regra, tumores unilaterais ${ }^{(4)}$.

Histologicamente, os melanomas de coróide podem ter três apresentações: fusiforme, clinicamente menos agressivos e de bom prognóstico; epitelióides, clinicamente mais agressivos e prognóstico desfavorável; ou ainda por celularidade mista, de prognóstico intermediário ${ }^{(2,4)}$. As metástases ocorrem por via hematogênica e o principal sítio é o fígado ${ }^{(5)}$. O prognóstico varia de acordo com o tamanho do tumor, sua localização intra- 
ocular, tipo histológico, extensão extra-ocular e idade no momento diagnóstico.

O diagnóstico precoce, feito através de cuidadoso exame oftalmológico e o tratamento adequado é fundamental para melhorar o prognóstico visual e de vida do paciente. Em nosso meio, não é raro a descoberta do tumor em estágio avançado, limitando as opções terapêuticas e aumentado a morbidade e a mortalidade pela patologia ${ }^{(6)}$.

Arcieri et al., (2002) observaram a queixa da redução da visão, como primeiro sintoma oftalmológico, em até $66,5 \%$ dos casos de melanoma de coróide ${ }^{(7)}$. Alguns pacientes podem ainda referir dor ou fotopsias como causa de procura do exame ocular $^{(3)}$. Destaca-se, porém, que as manifestações clínicas podem estar ausentes até crescimento importante da lesão, sendo fundamental a realização de exames oftalmológicos de rotina e freqüentes, incluindo a fundoscopia indireta sob midríase.

Ainda que existam várias opções terapêuticas, nenhuma delas garante cura ao paciente. A escolha do tratamento será baseada na possibilidade de manutenção da visão, do globo ocular afetado e na redução de metástases à distância ${ }^{(1)}$. O olho afetado pode ser observado, tratado de forma conservadora pela fotocoagulação, preferencialmente pela termoterapia transpupilar (TTT), radioterapia, em feixe ou por placa episcleral (braquiterapia). Ou ainda de forma cirúrgica pela ressecção local, enucleação ou exenteração, associando-se de forma adjuvante a quimioterapia ou imunoterapia sistêmica ${ }^{(7)}$. As opções de tratamento tornam-se menores quanto maior for o tumor. A enucleação do olho tem sido o tratamento tradicional ${ }^{(2-8)}$. Em geral, as taxas de sobrevivência por 10 anos após enucleação do olho afetado por melanoma de coróide são em média de $81,2 \%$ para tumores pequenos, $60,0 \%$ para tumores médios e $34,8 \%{ }^{(9)}$, porém outros fatores como tipo celular, volume do tumor, presença de disseminação extra-escleral e possibilidade de manipulação do olho durante a enucleação também estão relacionados com a sobrevida ${ }^{(10)}$. Em pacientes com doença metastática, o tempo médio de sobrevivência é menor do que um ano, a validade da quimioterapia é limitada e o tratamento para o olho afetado é apenas alívio sintomáti$\mathrm{Co}^{(2)}$. O acompanhamento dos pacientes com melanoma maligno de coróide precisa ser longo, pois as metástases podem ocorrer até 10 anos após o diagnóstico(2).

O objetivo do presente estudo é relatar as características dos pacientes diagnosticados e tratados com melanoma de coróide no Serviço de Oftalmologia do Hospital Regional de São José - Santa Catarina.

\section{MÉTODOS}

Entre fevereiro e outubro de 2003, analisou-se e descreveu-se os prontuários dos pacientes que entre março de 1997 e julho de 2003 tiveram o diagnóstico de melanoma de coróide e o tratamento para a patologia realizado no Serviço de Oftalmologia do Hospital Regional de São José - SC.

Todos os pacientes incluídos no estudo foram submetidos a anamnese oftalmológica e geral, sendo realizado exame ocular completo, com aferição acuidade visual, tonometria, biomicroscopia, oftalmoscopia sob midríase medicamentosa com mapeamento de retina e ultra-sonografia ocular. Após o diagnóstico clínico, para investigação de metástases à distância, os pacientes submeteram-se a avaliação sistêmica onde foi realizado hemograma, glicemia jejum, provas de função hepática (TGO e TGP) e renal (uréia e creatinina), realizado ultra-sonografia de abdome, raios $\mathrm{X}$ de tórax, tomografia computadorizada de crânio e órbita.

No presente estudo, a partir do prontuário destes pacientes foi pesquisado a idade, sexo, sinais e sintomas, métodos usados para diagnóstico, tipo e tamanho do tumor e tratamento realizado, conforme protocolo elaborado para melanoma maligno de coróide.

Pelo seu desenho descritivo e histórico, os pacientes não foram interrogados ou examinados, uma vez que os dados foram coletados a partir de seus prontuários. Estes não tiveram sua identidade revelada, estando cientes da possibilidade dos dados referentes à sua patologia serem utilizados em estudos científicos.

\section{RESULTADOS}

Foram incluídos no estudo 24 pacientes diagnosticados com melanoma maligno de coróide e que optaram por fazer tratamento no Serviço de Oftalmologia do Hospital Regional de São José (HRSJ) no período de $1^{\circ}$ de março de 1997 a 21 de julho de 2003.

Dos pacientes incluídos no estudo, $12(50 \%)$ eram do sexo masculino e 12 (50\%) eram do sexo feminino. Vinte $(83,33 \%)$ pacientes eram brancos, $3(12,5 \%)$ eram negros e $1(4,16 \%)$ era pardo. A idade dos pacientes no momento do diagnóstico

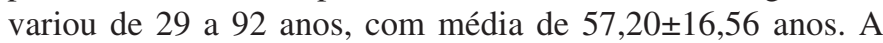
maioria dos pacientes (75\%) tinha entre 40 a 79 anos de idade no momento do diagnóstico, conforme gráfico 1 .

Todos os 24 pacientes (100\%) apresentaram tumores unilaterais, sendo que 17 (70,83\%) pacientes tiveram acometimento do olho esquerdo e $7(29,16 \%)$ do olho direito. A visão corrigida no olho afetado, no momento do diagnóstico, em

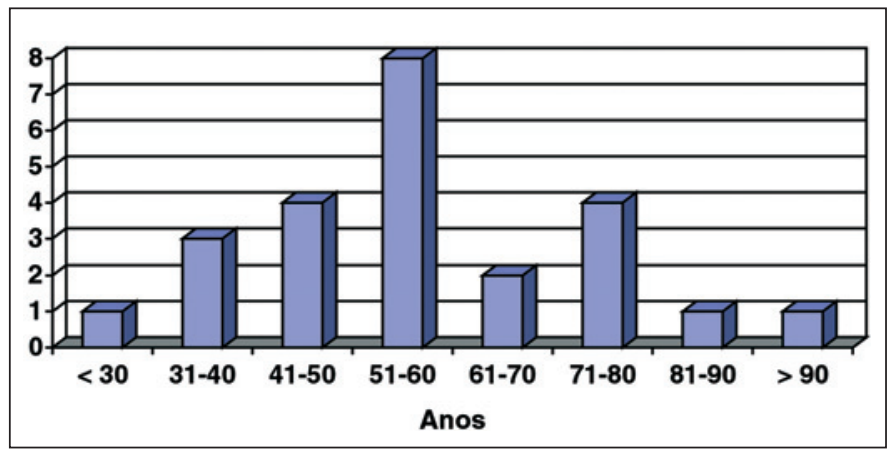

Gráfico1 - Distribuição da amostra quanto à faixa etária dos pacientes acometidos por melanoma de coróide. HRSJ, 2003 
$37,5 \%$ dos pacientes era apenas de percepção de vultos (movimento de mãos) (Gráfico 2).

A pressão intra-ocular do olho alterado, aferida através da tonometria de aplanação de Goldmann, nessa população variou entre 12 e $22 \mathrm{mmHg}$, sendo a PIO média de 16,41 $\$ 3,06 \mathrm{mmHg}$.

A queixa principal dos pacientes desta série, ao procurarem atendimento oftalmológico, foi a baixa da acuidade visual, presente em $20(83,33 \%)$ pacientes. Três $(12,5 \%)$ pacientes procuraram atendimento inicial devido à associação entre dor ocular e proptose, e $1(4,16 \%)$ queixou-se de dor ocular isolada. Entre o início dos sintomas e o atendimento no Serviço de Oftalmologia do HRSJ houve o intervalo de 7 dias a 1 ano, com média de 152,12 $\pm 114,00$ dias.

Quatorze $(58,33 \%)$ pacientes apresentaram descolamento de retina no olho acometido pelo tumor e $2(8,33 \%)$ comprometimento do nervo óptico. Na investigação clínica de rotina, realizada após o exame oftalmológico, em 2 (8,33\%) pacientes foi diagnosticada metástase à distância, sendo que um paciente apresentou metástase pulmonar e o outro para pele.

$\mathrm{Na}$ ultra-sonografia ocular, todos os 24 (100\%) pacientes apresentaram tumores classificados quanto ao tamanho como grandes segundo o "Collaborative Ocular Melanoma Study" ${ }^{(10)} \mathrm{com}$ diâmetro basal superior a $16 \mathrm{~mm}$ e/ou altura maior que $8 \mathrm{~mm}$. O tratamento cirúrgico aplicado em todos os casos foi a enucleação. Os dois $(8,33 \%)$ pacientes onde foi diagnosticada metástase à distância foram enucleados, como adjuvante do tratamento por quimioterapia.

O resultado do exame anátomo-patológico das peças cirúrgicas evidenciou que a maioria dos pacientes, com relação a celularidade, apresentou melanoma maligno de coróide do tipo células fusiformes $(58,33 \%)$ (Tabela 1). De acordo com o exame

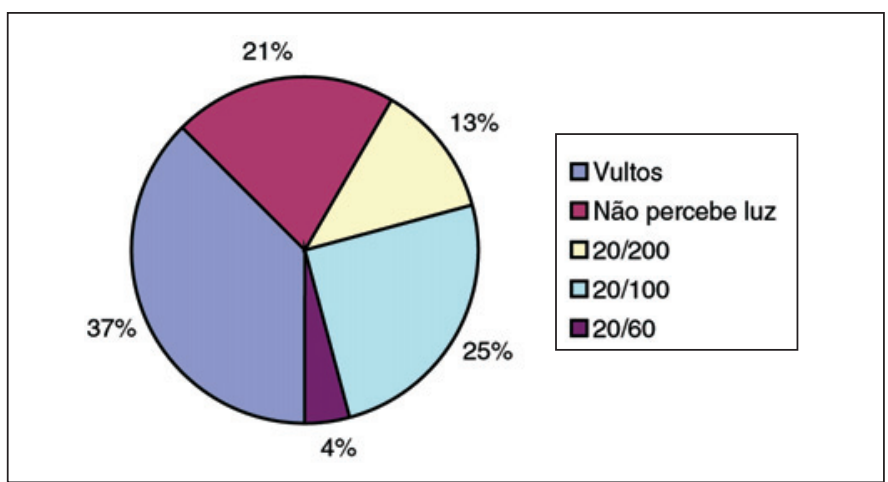

Gráfico 2 - Distribuição da amostra quanto à acuidade visual em olho acometido por melanoma de coróide. HRSJ, 2003

Tabela 1. Distribuição da amostra quanto à classificação histológica do melanoma maligno de coróide após exame anátomo-patológico da peça cirúrgica. HRSJ, 2003

\begin{tabular}{lcc|} 
Tipo histológico & Número & Porcentagem \\
Fusiformes & 14 & 58,33 \\
Epitelióides & 7 & 29,16 \\
Mistos & 3 & 12,50 \\
Total & 24 & 100,00 \\
\hline
\end{tabular}

anátomo-patológico das peças cirúrgicas, o tamanho dos tumores foi em média de $11,8 \pm 4,5 \mathrm{~mm}$ de diâmetro basal.

\section{DISCUSS $\tilde{A} O$}

Conforme havia sido previamente observado na literatura $^{(2,7,11-13)}$, no presente estudo não houve diferença em relação ao sexo em pacientes acometidos por melanoma maligno de coróide, sugerindo que a doença não tenha afinidade especial pelo sexo masculino ou feminino. A média de idade dos pacientes analisados foi de 57,20 $\pm 16,56$ anos, coincidindo com a faixa etária de maior acometimento do tumor ${ }^{(2-3,14)}$. Houve uma maior incidência do tumor em pacientes da raça branca $(83,33 \%)$, sendo este dado coincidente com os da literatu$\mathrm{ra}^{(1,13)}$. Porém foram relatados três $(12,5 \%)$ casos de pacientes negros, em confronto com a raridade desses tumores em pacientes dessa raça ${ }^{(1,13)}$.

Os melanomas malignos de coróide são por via de regra tumores unilaterais ${ }^{(2,4,7)}$ e os pacientes estudados acometidos pela neoplasia apresentaram essa característica.

Em concordância com observado por Arcieri et al. (2002), em estudo realizado em Uberlândia, onde a maioria dos diagnósti$\cos (66,5 \%)$ foram realizados devido a queixa de perda visual ${ }^{(7)}$, no presente estudo, a diminuição da visão estava presente em $20(83,33 \%)$ pacientes, sendo o sintoma inicial que fez com que os mesmos procurassem atendimento oftalmológico. Na literatura, em vários estudos ${ }^{(2,15)}$, muitos casos de melanoma de coróide são detectados na fase inicial da doença através de exames de rotina, estando os pacientes praticamente assintomáticos. No presente estudo, o tempo do início da sintomatologia até o atendimento inicial no Serviço de Oftalmologia do HRSJ foi em média de 152,12 $\pm 114,00$ dias. Nenhum dos pacientes do presente estudo foi diagnosticado quando ainda assintomático através de exames oftalmológicos de rotina, o que vem a demonstrar que todos eles foram diagnosticados em estágios avançados do tumor. Este demora no atendimento inicial desnuda uma realidade preocupante, como não existem campanhas de prevenção e pelo fato de, principalmente no interior do estado de Santa Catarina, a população menos favorecida ter dificuldades para o acesso ao atendimento oftalmológico, o encaminhamento ao HRSJ, que funciona como centro de referência é demorado, retardando o correto diagnóstico e tratamento adequado, com piora do prognóstico.

Todos os pacientes foram investigados primeiramente através da oftalmoscopia binocular indireta e ultra-sonografia, sendo este último fundamental para a confirmação diagnóstica. O ultra-som permite exame do segmento posterior do olho mesmo nos casos de opacidades dos meios oculares, possibilitando a identificação de massas sólidas e o conseqüente diagnóstico diferencial, além de permitir a aferição do tamanho aproximado do tumor ${ }^{(16-17)}$, influenciando não só na escolha do tratamento, bem como no prognóstico do paciente ${ }^{(8-10)}$.

Quando os tumores são diagnosticados tardiamente, os pacientes estão sujeitos a apresentarem descolamento de reti- 
na, invasão do nervo óptico, raramente extensão escleral, comprometimento macular além de metástases extra-oculares $^{(18)}$. Por todos os pacientes terem sido diagnosticados em estágios avançados do tumor, $14(58,33 \%)$ pacientes tiveram descolamento de retina e $2(8,33 \%)$ comprometimento do nervo óptico. Embora o principal sítio de metástase a distância desses tumores seja o fígado ${ }^{(5)}, 2(8,33 \%)$ pacientes apresentaram metástase extra-ocular sendo um comprometimento pulmonar e outro para a pele. Todos os pacientes após diagnóstico e tratamento foram encaminhados para serviço de oncologia clínica para acompanhamento.

Quanto à classificação histológica, no presente estudo, houve um predomínio do tipo histológico fusiforme $(58,33 \%)$, seguido pelo epitelióide $(29,16 \%)$ e de celularidade mista $(12,5 \%)$. Estes dados são divergentes com outros estudos, onde a celularidade mista foi mais freqüentemente encontra$\mathrm{da}^{(2,7,14-15,19)}$. Os tumores fusiformes normalmente são menos agressivos clinicamente, com o crescimento mais lento e menor prevalência de metástases à distância. No entanto os pacientes aqui analisados apresentavam massas tumorais grandes, com um tamanho médio de $11,8 \pm 4,5 \mathrm{~mm}$ no maior diâmetro basal, denotando evolução de longa data, o que torna o prognóstico destes pacientes mais reservados, mesmo em tumores fusiformes.

A conduta em relação aos melanomas malignos de coróide é bastante controversa, no entanto, baseado na classificação de COMS, sugere-se a seguinte conduta em relação a esses tumores $^{(19)}$ :

1. Paciente sem qualquer sinal de doença metastática:

- Tumores de até $3 \mathrm{~mm}$ de espessura: observação periódica;

- Tumores de 3 a $8 \mathrm{~mm}$ de espessura e base até $15 \mathrm{~mm}$ : braquiterapia;

- Tumores com mais de $8 \mathrm{~mm}$ de espessura: indicado enucleação;

- Se base de até $15 \mathrm{~mm}$ : ainda pode-se tentar braquiterapia (prognóstico reservado);

- Se a base for maior que $15 \mathrm{~mm}$ : indicado enucleação.

2. Paciente com sinal de doença metastática: quimioterapia e enucleação se o olho for doloroso.

Como demonstrado, na escolha da opção terapêutica o tamanho do tumor é fundamental, no presente estudo todos os pacientes foram enucleados. No Brasil, a enucleação continua sendo a forma de tratamento mais empregada, devido ao menor custo, dificuldade de acesso a grandes centros com serviço de braquiterapia e fundamentalmente um diagnóstico mais tardio, pois ainda que existam casos onde o crescimento é rápido, geralmente um melanoma de coróide leva cerca de 5 anos para atingir grandes dimensões (base maior que $15 \mathrm{~mm})^{(20)}$. No grupo estudado todos os pacientes foram enucleados, inclusive os dois pacientes com diagnóstico de metástase à distância, como adjuvante a quimioterapia, conforme orientação do oncologista clínico, no entanto este tratamento é controverso e segundo alguns autores não aumentaria a sobrevida dos pacientes ${ }^{(9)}$.

Como qualquer neoplasia, o diagnóstico precoce dos me- lanomas malignos de coróide é fundamental. A situação social, econômica e cultural dos pacientes diagnosticados com melanoma de coróide no Brasil, caracterizada pela desinformação e até mesmo à dificuldade de atendimento contribui para o diagnóstico da lesão em fase avançada ${ }^{(6)}$. Nesta fase, as opções terapêuticas são mais limitadas, piorando o prognóstico dos pacientes, diminuindo a sobrevida.

Apesar da raridade ${ }^{(5,19)}$, mas diante da gravidade desse tumor, deve ser considerado o valor da oftalmoscopia indireta como prevenção e diagnóstico, bem como a necessidade de um esclarecimento da população em relação à significância das alterações oculares e visuais, pois, na grande maioria dos casos, é suficiente para detectar precocemente o tumor, melhorando o prognóstico e ampliando as opções terapêuticas. A informação através de campanhas preventivas, um melhor acesso à população em geral aos serviços públicos de saúde, junto à realização da oftalmoscopia binocular indireta pelo oftalmologista anualmente a partir dos 40 anos de idade são necessários para diagnosticar precocemente esses tumores, melhorando o prognóstico visual e sistêmico dos pacientes com melanoma maligno de coróide.

\section{CONCLUSÕES}

Os pacientes diagnosticados com melanoma maligno de coróide são na sua grande maioria brancos, com idade variando entre 40 a 79 anos, sendo que a sexta década de vida é a mais afetada. Não foi observada diferença entre os sexos e todos os tumores foram unilaterais. A grande maioria $(83,33 \%)$ dos pacientes procurou atendimento inicial devido à queixa de perda visual. O tempo de procura desses pacientes do início

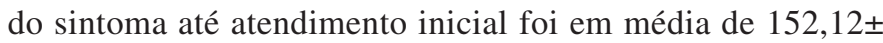
114,00 dias. No presente estudo, todos os 24 pacientes foram diagnosticados em estágios tardios do tumor, fato que piora o prognóstico visual e sistêmico além de limitar as opções terapêuticas. O tipo histológico fusiforme, caracteristicamente de menor agressividade, esteve presente em 58,5\% dos casos, porém todos os tumores foram classificados como grandes segundo o "Collaborative Ocular Melanoma Study"(10), com tamanho médio de $11,8 \pm 4,5 \mathrm{~mm}$ de maior diâmetro basal, sendo devido a isto, todos eles submetidos a enucleação para tratamento da neoplasia.

\section{ABSTRACT}

Purpose: To describe the characteristics of patients choroidal melanoma diagnosed and treated at the Ophthalmology Unit of the Regional Hospital of São José - Santa Catarina, and to show the results. Methods: Analysis of 24 medical records of patients with a diagnosis of malignant choroidal melanoma from, March $1^{\text {st }} 1997$ to July 21 ${ }^{\text {st }} 2003$, regarding: age, gender, race, symptoms and duration, diagnostic methods, performed treatment, size and type of tumor. Results: There was no 
difference regarding gender, and the average age of patients was about $57.2 \pm 16.56$ years. The tumor predominated in white people $(83.33 \%)$ and low visual acuity was the most frequent symptom $(83.33 \%)$. The average time from the beginning of symptomalogy up to the initial treatment was $152.12 \pm 114.00$ days. Indirect ophtalmoscopy and ultrasound were performed in all the patients in order to diagnose the tumor. All patients underwent enucleation, because they showed large tumors (greatest basal diameter higher than $16 \mathrm{~mm}$ at to USG). The most frequent type was that of fusiform cells $(58.5 \%)$ and the average size of the tumors was $11.8 \pm 4.5 \mathrm{~mm}$ basal diameter. Two patients presented systemic metastasis. Conclusions: All patients were diagnosed when the tumor was already advanced, making the visual and systemic prognostic worse and reducing the therapeutic options, and therefore were enucleated.

Keywords: Melanoma/diagnosis; Choroidal neoplasms; Eye enucleation

\section{REFERÊNCIAS}

1. Hodge WG. Trato uveal e esclera. In: Vaughan D, Asbury T, Riordan-Eva P (eds). Oftalmologia geral. 4a ed. São Paulo: Ateneu; 1998, p.159-60.

2. Melgares Ramos MA, Santos Silva D, Puig Mora M, Cruz Mojarrieta J, Mesa Zarate E, Cordies Justín N. Melanoma de coroides: estudio de la casuística en el INOR en el período de 1980-1996. Rev Cuba Oncol. 1998;14(3):149-54.

3. Augsburger JJ, Shields CL, Shields JA. Tumores oculares. In: Rhee DJ, Pyfer MF (eds.). Manual das doenças oculares "Wills Eye Hospital". Diagnóstico e tratamento emergencial das doenças oculares. 3a ed. Rio de Janeiro: Cultura Médica; 2002. p.195-8.

4. Shields C, Shields JA. Introduction to melanocytic tumors of the uvea. In: Shields CL, Shields JA. Intraocular tumors: a text and atlas. Philadelphia: WB Saunders; 1992. p.45-59.
5. Fay A, Jakobiec FA. Doenças do sistema ocular. In: Goldman L, Bennett JC (eds). Cecil tratado de medicina interna. 21a ed. Rio de Janeiro: Guanabara Koogan; 2001. p.2485.

6. Caramelli CB, Rotenberg M, Avakian A, Silva ALB, Damico FM, Takei LM, Onclinx T. Tumores oculares: fatores de atraso no atendimento oftalmológico. Arq Bras Oftalmol. 1997;60(6):604-7.

7. Arcieri ES, Fonseca D, França ET, Braga EF, Ferreira MA. Estudo de melanoma de coróide na Universidade Federal de Uberlândia. Arq Bras Oftalmol. 2002; 65(1):89-93.

8. Fraundefer FT, Boozman FW, Wilson RS, Thomas AH. No-touch technique for intraocular malignant melanomas. Arch Ophthalmol. 1977;95(9):1616-20.

9. Bell DJ, Wilson MW. Choroidal melanoma: natural history and management options. Cancer Control. 2004;11(5):296-303.

10. Santo RM, Bechara SJ. Tumores intra-oculares. Arq Bras Oftalmol. 1998;61 (2):242-55.

11. Baldo S, Kodjikian L, Belli F, Mariani F, Malvezzi E, Meeus P, et al. [Liver metastases of choroid melanoma. Retrospective study of 63 cases]. Tumori. 2003;89(4 Suppl):229-30. Review. Italian.

12. Straatsma BR, Diener-West M, Caldwell R, Engstrom RE. Collaborative Ocular Melanoma Study Group. Mortality after deferral of treatment or no treatment for choroidal melanoma. Am J Ophthalmol. 2003;136(1):47-54.

13. López-Caballero C, Saornil-Alvarez MA, Blanco-Mateos G, Frutos-Baraja JM, López-Lara F, González-Sansegundo C. Melanoma de coroides en melanosis óculi. Arch Soc Esp Oftalmol. 2003;78(2):99-102.

14. Albert D, Syed N. Cancer Committee, College of American Pathologists. Protocol for the examination of specimens from patients with uveal melanoma: a basis for checklists. Arch Pathol Lab Med. 2001;125(9):1177-82.

15. Romero JM, Finger PT, Rosen RB, Lezzi R. Three-dimensional ultrasound for the measurement of choroidal melanomas. Arch Ophthalmol. 2001;119(9): 1275-82.

16. Robertson DM. Melanoma endoresection: a perspective. Retina. 2001;21(5): 403-7. Review.

17. Lee DS, Anderson SF, Perez EM, Townsend JC. Amelanotic choroidal nevus and melanoma: cytology, tumor size, and pigmentation as prognostic indicators. Optom Vis Sci. 2001;78(7):483-91.

18. Erwenne CM. Os métodos de tratamento e as rotinas em tumores intra-oculares. Arq Bras Oftalmol. 1998;61(5):602-7.

19. Sahel JA, Pesavento R, Frederick AR Jr, Albert DM. Melanoma arising de novo over a 16-month period. Arch Ophthalmol. 1988;106(3):381-5.

20. Zimmerman LE, McLean IW. An evaluation of enucleation in the management of uveal melanomas. Am J Ophtalmol. 1979;87(6):741-60. Review. 